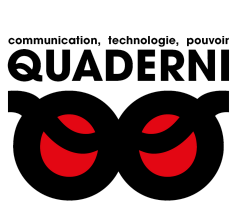

\title{
Quaderni
}

Communication, technologies, pouvoir

95 | Hiver 2017-2018

Logiques numériques des radicalisations

\section{Figures du Chef Cuisinier}

Seconde partie : d'une autorité civique revendiquée aux engagements citoyens et collaboratifs des "mangeurs"

Dominique Pagès

\section{(2) OpenEdition \\ 1 Journals}

Édition électronique

URL : https://journals.openedition.org/quaderni/1149

DOI : 10.4000/quaderni.1149

ISSN : 2105-2956

Éditeur

Les éditions de la Maison des sciences de l'Homme

Édition imprimée

Date de publication : 5 février 2018

Pagination : 95-114

Référence électronique

Dominique Pagès, «Figures du Chef Cuisinier », Quaderni [En ligne], 95 | Hiver 2017-2018, mis en ligne le 05 février 2020, consulté le 03 septembre 2021. URL : http://journals.openedition.org/quaderni/

1149 ; DOI : https://doi.org/10.4000/quaderni.1149 


\section{Communication}

\section{Figures du \\ Chef Cuisinier Seconde partie :}

d'une autorité civique revendiquée aux engagements citoyens et collaboratifs des "mangeurs"

De la gastro-diplomatie à l'engagement civique et public dans le devenir des villes, le 'grand' chef cuisinier redessine sa place et son rôle symboliques 'autrement'. En s'inscrivant dans les stratégies et politiques publiques contemporaines autour de l'alimentation et des patrimoines culinaires, en se positionnant au cœur des débats sur la transition alimentaire et la refondation des systèmes alimentaires, il joue une partie complexe : cultiver sa notoriété et soigner son personal branding tout en participant à la recomposition d'une société autour du développement durable ; s'emparer des causes planétaires tout en restant culinairement crédible ${ }^{1}$.

La diversité des figures médiatiques du chef cuisinier (envisagée dans le précédent article ${ }^{2}$ ) nous est rapidement apparue de surface, chacune d'entre elles contribuant à la vision d'un chef engagé et 'sauveur', d'un chef mettant sa reconnaissance sociale au service d'un nombre croissant de causes. Une héroïsation chatoyante mais néanmoins ambivalente qui en ferait donc, à une époque de crises et de peurs plus ou moins fantasmées, une instance 'autorisée', crédible si ce n'est légitime, pour nous inviter au dévelop-

Dominique Pagès

MCF CELSA - Paris Sorbonne, Laboratoire GRIPIC et Food LAB. 2.0 pement durable de nos systèmes alimentaires. Partout dans le monde, les chefs se saisissent, à bras le corps, des problématiques sociales et environnementales, écologiques et culturelles, inhérentes aux crises et mutations alimentaires contemporaines. Ils se font médiateurs, intermédiaires, passeurs requalifiant, hiérarchisant et recontextualisant les enjeux planétaires de l'alimentation. En déployant leurs actions à l'échelle locale (celle du quotidien des communautés), ils permettent le passage de prises de positions macro-politiques à des actions 
concrètes et tangibles, revendiquées et clairement inscrites dans des stratégies territoriales volontaristes et participatives, c'est-à-dire susceptibles de prendre en considération la capacité de création, d'innovation, d'imagination des mangeurs.

Dans ce second article, après avoir questionné plus avant cette célébration d'un chef tout à la fois " garant », « leader charismatique » et « acteur responsable » (et plus encore lanceur d'alerte), nous interrogerons la pertinence de ces mises en scène éthiques au regard des imaginaires et des pratiques alimentaires. Puis, nous reviendrons sur cette exemplarité spectacularisée du chef, cette mise en récit en vue d'inciter et d'être imitée, de susciter des identifications et d'encourager à l'action (du global au local et au terroir), avant d'envisager le « chef de demain », au fil de quelques récentes représentations. Ainsi, les enjeux et l'opérativité de son influence seront questionnés plus avant.

\section{L'influence mise en scène : plurielle et contra- dictoire?}

La seule évocation de la figure du chef suscite chez chacun des images fortes, émotionnelles, rassurantes, positives, bienveillantes. De là, sans doute, la surexploitation médiatique et publique de ce pouvoir d'évocation et d'incarnation, le chef rendant tangibles des connaissances et des problématiques complexes (liées à des recherches scientifiques difficiles à vulgariser). Si le chef peut s'instituer porte-parole de la diversité des causes précédemment évoquées, c'est aussi parce qu'à une époque de perte de confiance et de repères ou de doutes, il donne l'exemple, celui d'un homme qui s'engage, accepte le changement, s'y coltine et prend en charge la destinée alimentaire du monde.

\section{Le chef, "un garant"}

Cautionnant les recherches, vulgarisant les savoirs, rendant proches les problématiques publiques, il reste bien une figure de l'autorité alimentaire, mais une figure moins ostentatoire et frontale qu'elle ne le fut : nombre de recherches nous ont progressivement montré, ces dernières décennies, combien l'autorité est un processus qui se fragilise et qu'il s'agit de relégitimer et de redéfinir sans cesse. Famille, école, monde $\mathrm{du}$ travail, institutions ainsi que la plupart des instances traditionnelles d'autorité connaissent un déficit croissant de légitimité, laissant la place (du moins apparemment, médiatiquement) à des figures inédites mais familières (stars et people dont les engagements font les unes des magazines, voire des J.T.).

Si le chef devient un garant, c'est qu'il rassure et oriente à un moment où se télescopent malentendus et incompréhensions, rumeurs et peurs, méconnaissances et croyances (parfois sectaires) autour de l'alimentation. Son 'autorité' est moins celle d'un homme de pouvoir (agissant sur les normes et les règlements) que celle d'un homme informé et compétent (des compétences ici narratives, pragmatiques et relationnelles). Et cela même si certains chefs se font aussi garants pour des marques industrielles qu'ils dénoncent sur d'autres scènes : Paul Bocuse avec William Saurin, Joël Robuchon avec Fleury Michon (ou d'autres marques, hôtelières, de fabrication de meubles, de parcs d'attraction), etc. 
Les chefs étoilés s'inscrivent dans des stratégies de communication publique en tant que relais et recours, à un moment où la parole publique est quelque peu décrédibilisée mais, paradoxalement, où son travail idéologique pour faciliter la cohésion et la mobilisation sociales (autour des questions alimentaires et de développement durable) devient pourtant plus que jamais nécessaire.

Les pouvoirs publics semblent envisager les chefs comme des gate keeper, non seulement pour la défense de la gastronomie et des patrimoines culinaires mais aussi pour la transition alimentaire : ceux-ci, affirmant haut et fort leur autorité présumée sur les choix alimentaires et étant supposés capables de « faire passer » des enjeux souvent perçus comme abstraits ou experts (sanitaires, de prévention, agricoles, écologiques...), se voient régulièrement invités à prendre la parole dans les conférences citoyennes, les débats publics, les États généraux de l'alimentation, pour faire bouger les lignes et signifier concrètement les mutations. Ainsi, sont-ils aussi associés aux actions d'éducation et de bien-être, de tourisme et de culture, de valorisation des produits alimentaires.

Pour rappel, le gate keeper est cet intermédiaire chargé d'ouvrir l'accès à certaines informations et données via la médiatisation et les médiations ; il laisse entrer certaines informations dans le champ public et en bloque d'autres; jouant le rôle de filtre sélectif attirant l'attention des publics, il cherche à orienter les représentations, les opinions, les comportements. Le chef, en re-sacralisant par ses interventions publiques la nature, le terroir, la terre, du local à la planète, élargit donc son champ d'expertise et s'inscrit (discursive- ment) dans l'arène publique et marchande autour de l'alimentation : il est supposé réinstaurer une confiance (le ‘bonheur alimentaire' n'existe qu'à cette condition), remettre « dans le bon sens » nos relations aux aliments, aux produits alimentaires, aiguiller nos comportements et nos pratiques alimentaires, un peu comme un coach le ferait... et le fait, télévisuellement (évolution dans ce sens des émissions culinaires).

Certains y voient une stratégie avant tout opportuniste, d'adaptation aux valeurs contemporaines (naturalité, santé, écologie, frugalité...). L'opportunisme, qui consiste à tirer le meilleur parti des circonstances, serait donc le signe d'une capacité d'adaptation des chefs aux circonstances, à suivre les processus de changement de notre société (qui voit se réorganiser et se transformer nos pratiques alimentaires). Mais l'enjeu est autrement plus complexe : l'invitation récente (septembre 2017) à un déjeuner à l'Élysée faite par le président Macron aux 180 chefs gravitant autour des Bocuses d'or (concours mondial de la gastronomie) en témoigne. Les propos du Président qui, à cette occasion, les invita à prendre part aux États généraux de l'alimentation, relativisent cette interprétation tactique : «Le déjeuner d'aujourd'hui prouve à ceux qui n'étaient pas encore convaincus que la nourriture est bien une affaire d'État (...). La France est attendue pour sa gastronomie. Elle rayonne par sa gastronomie et ce que les étoilés représentent lorsqu'ils accueillent le monde dans notre pays, ou lorsqu'ils essaiment le savoir français par delà nos frontières $»^{3}$. Le chef étoilé est bel et bien construit comme l' " ambassadeur »d'une gastro-diplomatie dont les enjeux et les règles changent. Mais, pour autant, sont-ils, au quotidien, audibles par les mangeurs ? 


\section{Le chef, " un leader charismatique "}

La communication publique est censée organiser et influencer la croyance et la confiance en la collectivité identitaire ; de là, sa dimension parfois affective et incarnée, son appel à des figures relais (positives et créatives). Il ne s'agit pas seulement pour elle de faire connaître (l'information), de faire comprendre (l'éducation) mais aussi de faire adhérer (séduire, émouvoir, enchanter...).

Ainsi l'ethos du 'chef garant', qui se concrétise par des discours (textuels, visuels, gestuels, comportementaux) volontaires, cherche à susciter la confiance mais aussi la réassurance : certitude des postures (bras croisés, franchise du port et corps tonique en mouvement, aura de faiseur de miracles ou de magicien), assurance des regards, francs et déterminés, disponibilité (à l'écoute, enthousiaste) ; invitations cordiales et fermes à le rejoindre (par un geste d'appel, une interpellation concrète) ; intégration à des collectifs « résolus » (importance croissante des images de groupes compacts de chefs, signifiant la détermination commune).

Cette autorité médiatisée des chefs cuisiniers dépasse donc la seule confiance culinaire et la seule garantie alimentaire. En sortant de la cuisine, en revêtant les atours du sauveur, en s'engageant tous azimuts, le chef revêt les habits du leader charismatique, joue sur les ressorts du charisme, cherchant à susciter l'adhésion, la mobilisation voire la vocation; il cherche à agir tant sur les idéaux et les valeurs, les attitudes et les opinions que sur les conduites et les comportements (voir les communications précédemment évoqués des chefs Alain Ducasse, Jamie Oliver,
Olivier Roellinger, Thierry Marx).

L'émergence d'une figure et d'un mouvement charismatiques signifie une crise, un désordre, la nécessité d'un changement face à une impasse ou à un discrédit des 'responsables', elle tend vers la possibilité d'un 'ordre naissant' : en se positionnant entre un passé et un futur, un monde ancien et un autre en émergence, le chef se positionne comme une issue possible, l'annonciateur d'un passage vers un autre système alimentaire. Par sa chaleur communicative, par son intelligence émotionnelle et sa capacité à inspirer la confiance, il peut susciter adhésion et mobilisation vers de nouvelles valeurs, de nouvelles manières de vivre alimentaires. Ses gestes aguerris, ses mots simples et clairs, son énergie positive sont autant de médiations pour annoncer un ordre nouveau et incarner de nouveaux crédos. Le chef se met en scène comme signe d'identification, emblème d'une société plus responsable, donnant l'illusion de donner accès à un idéal. Mais le charisme doit correspondre à un besoin effectif d'identification de la part d'une opinion ou d'une communauté qui seraient en attente ${ }^{4}$, ce qui, aujourd'hui, n'est pas une évidence.

\section{De l'autorité à l'influence : figures implicites de la mobilisation}

Le chef médiatique chercherait à influer, à susciter des changements de mentalités et de comportements, des croyances et des identifications autour de nouvelles valeurs alimentaires. Les processus d'influence convoqués semblent multiples, tout comme les publics interpellés (du grand public à tous les acteurs de la chaîne alimentaire, des élèves cuisiniers aux étudiants 
en géographie ou histoire alimentaires, des plus jeunes aux plus âgés, des sportifs aux malades...).

$\mathrm{Au}$ fil des communications envisagées dans le premier article, les chefs s'appuient sur des processus d'influence divers, alliant conviction et séduction, adresse individuelle et collective, jouant (tour à tour ou selon les médias) tant sur l'imitation simple (suivisme des modèles proposés par souci de rester dans le groupe, à l'exemple du chef Raymond Oliver) ; sur l'identification collective (adhésion plus émotionnelle aux valeurs communes, à l'exemple de son fils Michel ou de Jamie Oliver) ; sur l'intériorisation (adhésion individuelle, soucieuse de se fonder sur un jugement propre, une conviction intime avant d'accepter idées, valeurs, normes, à l'exemple des chefs René Redzepi, Pierre Gagnaire ou Cyril Ligniac) ; sur l'engagement collectif (appropriation autonome et action, émancipation de la tutelle, à l'exemple de Thierry Marx et de divers jeunes chefs).

Le chef serait donc devenu l'un de ces nouveaux acteurs 'talentueux' qui tentent d'inventer une nouvelle culture alimentaire pour le $\mathrm{XXI}^{\mathrm{e}}$ siècle, favorisant le développement d'une société plus responsable et durable, inclusive et porteuse de sens. Son autorité affichée (originellement liée à un savoir-faire) évoluerait bel et bien vers des compétences et des savoirs élargis mais aussi vers une capacité d'anticipation et de prédiction (le talent comme légitimité et plus encore comme autorité). Il pourrait même, par certains côtés, s'assimiler parfois à celle d'un « lanceur d'alerte » : ainsi ces chefs qui défendent l'agriculture bas carbone, développent une cuisine bas-carbone, calculent le poids carbone des plats... En se mettant au diapason d'une cuisine responsable, ils invitent à faire évoluer les croyances alimentaires, à de nouveaux répertoires d'action, à remettre en question nos habitudes incorporées (celles qui structurent nos pratiques alimentaires). Mais ces récits bien huilés et omniprésents sont-ils pour autant en phase avec les évolutions des imaginaires, comportements et pratiques alimentaires contemporains ? À une époque où les consommateurs sont de plus en plus défiants mais aussi mieux informés sur les grands problèmes alimentaires, plus sensibilisés aux effets de leurs pratiques alimentaires, quelle place le chef peut-il vraiment légitimement prendre ?... Il n'y a d'autorité que reconnue par ceux sur lesquels elle s'exerce.

Cet ethos affiché est-il en phase avec les mentalités, les imaginaires actuels, notamment ceux des plus jeunes? Cet ethos mis en images qui attribue au destinataire (le citoyen mangeur) une certaine place est-il en phase avec les savoirs sur les mangeurs? Qui sont ces mangeurs qu'il faudrait éduquer, évangéliser, mobiliser ? Seraient-ils si aveuglés, déboussolés et démunis que pourraient le laisser croire les interpellations des chefs ? À qui parle le chef?

\section{D'une figure l'autre : un mangeur paradoxal et «branché »}

« Si les consommateurs sont irrationnels, il est encore plus irrationnel de ne pas étudier comment ils raisonnent », Claude Fischler.

Depuis une vingtaine d'années, les travaux sur les imaginaires, les comportements et les pratiques alimentaires se diversifient : sociologues, 
anthropologues, sémiologues, psychologues... tentent de comprendre ce que manger veut dire aujourd'hui, de saisir les mutations culturelles de l'alimentation contemporaine mais aussi de comprendre les prises de décision des mangeurs, leurs mécanismes et processus.

\section{Un mangeur observé}

Des chercheurs comme Claude Fischler, Jean Pierre Poulain, François Ascher, Jean Pierre Corbeau ont ainsi renouvelé les savoirs, rebattu les cartes des connaissances ${ }^{5}$. De leurs travaux (dont nombre ont été relayés par l'OCHA ${ }^{6}$ ), nous pouvons rappeler quelques apports déterminants pour saisir l'actualité. Les mangeurs contemporains souffriraient, selon eux, de plusieurs maux qui se répondraient et s'actualiseraient via des comportements singuliers. Ainsi, celui de la 'gastro-anomie' (l'absence de règles relatives à ce qui se mange) qui semble le mal le plus central car traduisant à la fois une profonde dérégulation sociale, une déstructuration des repas, une individualisation des comportements... et éclairant ainsi certains excès actuels, très contradictoires :

- ceux des régimes en tout genre : les régimes de santé (qui répondent à des allergies, des carences, des intolérances avérées) ; les régimes 'sans' (sans gluten, sans lactose, sans viande ni produits animaux...), très fortement normés mais pas nécessairement fondés sur un diagnostic médical ; les régimes 'éthiques' ou 'spirituels' (végétarisme, véganisme, macro-biotique) qui peuvent témoigner d'une critique sociale; ainsi que les régimes de tous genres, 'sur mesure', cherchant à traiter et résoudre de véritables phobies alimentaires, individuelles ou collec- tives (communautés avo-lacto-végétariennes, pesco-végératiennes, etc.), au risque parfois de l'orthorexie (ensemble de pratiques alimentaires caractérisé par la volonté obsessionnelle d'ingérer une nourriture saine et le rejet systématique des aliments perçus comme malsains)... Autant de régimes dont certains peuvent apparaitre comme des dénis ou des résistances à la déstructuration des repas, à leur informalisation: Claude Fischler a ainsi finement étudié cette montée des alimentations particulières;

- ceux du grignotage permanent, lui aussi lié à l'individualisation des modes de vie, à des vies devenues plus mobiles (manger partout et n'importe quand, en marchant, dans le bus, dans le métro, dans la rue) ; grignotages qui participeraient d'une 'désintégration' des cadres spatiaux et temporels des repas (tout comme les plats réchauffés en solos, les plats auto-chauffants, mangés à toute heure) et d'un jeu (conscient ou pas) avec les normes et les règles, à rebours, des régimes juste évoqués ;

- ceux de la nostalgie inconditionnelle : la quête plurielle de produits oubliés ou disparus, de plats tombés en désuétude, d'un âge d'or de produits alimentaires 'naturels' et 'authentiques' apparaît comme une réaction classique aux changements et aux crises liées aux 'progrès' scientifiques et techniques. Nostalgie reposant sur une vision édénique de la ruralité bien singulière car jamais, semble-t-il, les risques n'ont été aussi faibles dans les pays développés ${ }^{7}$;

- ceux mis en scène par le Food Porn (représenter visuellement ce que l'on mange ou cuisine de manière à exciter, provoquer des sentiments 
de plaisir, au bord de l'obscène), relayé par les réseaux sociaux : manger quand je veux, ce que je veux, comme je veux, jusqu'à la goinfrerie et en me mettant en scène. Quand on tape «mangeur » (Google image, septembre 2017), une bonne partie des imageries qui apparaissent dans les premières pages sidère : photos de goinfres en situation (souvent des hommes jeunes) qui s'adonnent allègrement à des excès alimentaires de tout ordre (avaler le plus gros ou le plus haut hamburger, le plus grand nombre de saucisses en une fois, etc.) ; tristes clichés de bouches trop pleines, de ventres-gouffres pris en plongée et contreplongée... Ces images de l'excès (issues de blogs, de réseaux sociaux ou d'articles sur le food porn) témoignent d'un volontarisme performatif, d'une forme abrupte de conjuration, visant peut-être à fragiliser symboliquement voire à transgresser les normes, les règles et les standards traditionnels qui président aux choix conventionnels. D'autres images (sur 'l'anorexie' ou sur le 'corps parfait' notamment) leur font discrètement contrepoint.

Donc, d'emblée, un doute s'installe : ces mangeurs qui entrent en résistance et ont construit leurs positions et leurs postures au fil de lectures, de recherches, de consultations d'internet, des mangeurs qui s'engagent dans des communautés dont l'éclectisme mériterait de fines analyses, ne semblent pas être ceux auxquels s'adressent les chefs.

\section{Le bouleversement des pratiques alimentaires}

Le mangeur contemporain serait donc anxieux, face aux crises et aux risques (réels ou fantasmés) que les médias relatent en continu (de la vache folle aux pesticides ou additifs interdits) et face à sa propre responsabilité : que manger pour ne pas s'empoisonner? Comment associer au mieux les aliments ? Quels produits choisir ?... De là, pour les plus vigilants, une quête croissante d'informations et de données : le mangeur se fait réflexif, analyse les produits ainsi que son histoire alimentaire, envisageant la connaissance de celleci comme un levier pour une meilleure maîtrise de ses choix et de son devenir alimentaires. $\mathrm{S}$ 'informer, c'est pour lui résister aux rumeurs, ne pas s'engouffrer dans les peurs alimentaires, se défier des logiques publicitaires, ne pas «s'en laisser conter ». Cette quête d'informations s'alimente à une diversité croissante de sources et de circuits d'information : conseils et données fournis directement par les nutritionnistes, les naturopathes, les diététiciens, les militants écologistes, les scientifiques (dont les expertises ne sont pas toujours accessibles) mais aussi, indirectement, par les médias publics et marchands, les réseaux sociaux où se déploient une grande diversité de lanceurs d'alerte, plus ou moins légitimes. Le mangeur est donc au cœur d'un réseau de discours souvent pléthoriques et relativement contradictoires voire déroutants. Aujourd'hui, le rôle de l'information dans la socialisation alimentaire (processus d'appropriation de normes et de valeurs dans un cadre socialement et culturellement situé) n'est donc pas simple. Manger apparaît à bon nombre comme une pratique incertaine, tissée de suspicions envers les prescriptions industrielles mais aussi envers les informations et prescriptions publiques (souvent abstraites et culpabilisantes).

\section{Mangeurs des villes...}

Pour autant, les comportements évoluent car les questions alimentaires rejoignent aussi celles du 
bien-être et du plaisir corporels qui prennent le pas sur les seuls enjeux nutritionnels... De là, pour les plus fortunés, un refus de la fast food au bénéfice de la fast good, de la bistronomie et de nouveaux concepts 'vertueux' de restauration... et, pour le plus grand nombre, une redécouverte de la commensalité et des moments gourmands festifs, au fil de rencontres autour d'un plat, entre amis et voisins (et parfois visiteurs), à l'occasion de repas de rue, de pique-niques urbains ou de banquets de quartier (notamment grâce aux invitations des mairies). Si les mangeurs des villes ont perdu le contact avec la culture des produits agricoles et l'élevage des animaux, si les modèles alimentaires en place s'érodent, il semble néanmoins que 'faire table commune' redevienne plus quotidien et que la déstructuration des repas et leur informalisation soient compensées par un retour vers le collectif (illustré par la profusion des cours de cuisines, des ateliers de dégustation, des voyages gourmands). Le mangeur contemporain serait ainsi capable de mobiliser plusieurs formes de rationalités.

Ainsi en est-il pour le mangeur 'urbain'. Curieux et documenté, cherchant à réduire les risques mais aussi en quête de diversité et de découvertes, il redéfinit en termes de 'consommation réfléchie' ses pratiques, traquant additifs et sources diverses de pollution, multipliant ses lieux de ravitaillement (classiques, marchés bio, coopératives reliées aux circuits courts, boutiques ethniques, etc.) et ceux de sa consommation (bistrots, fast casual, bars à soupe, food trucks, ateliers burgers, coffee shops, etc.), fragilisant ainsi les lieux traditionnels de la distribution et de la consommation. Inconstant voire capricieux, le foodista urbain entre dans le jeu des rivalités des enseignes et déstabilise les comportements 'attendus' par les acteurs marchands dont la capacité d'adaptation semble, par ailleurs, de plus en plus rapide.

Si le chef devient pour le citadin synonyme de confiance (fondée sur la connaissance familière), de bien-vivre culinaire en ville, d'hyper-choix qualitatifs, de renouveau des styles de vie alimentaires, sa visibilité et sa lisibilité se diluent quelque peu dans une offre exponentielle et inconstante, soumise à une diversification des critiques. Acteur du quotidien des mangeurs des villes, cultivant la mise en scène de sa créativité, il incarne la food culture, nouvelle mythologie urbaine fondée sur l'inventivité tant de l'offre que des usages (voir les Food studies anglosaxonnes) ; plus obsédé pour bon nombre par la qualité des produits (nécessairement locaux) que par la virtuosité culinaire, œuvrant dans un lieu 'designé' (du plus frugal au plus ludique), le chef devient pour le mangeur un acteur culturel à part entière. Pour ce mangeur des grandes villes, il semble donc être moins le révolutionnaire évoqué précédemment que le médiateur d'une nouvelle idée de la gastronomie et de la consommation alimentaire, qu'un passeur de tendances et l'évangélisateur d'une nouvelle idée du goût ${ }^{8}$.

\section{Le mangeur connecté : pour le pire et le meilleur?}

L'extension des médiations numériques dédiées à l'alimentation, l'importance de « l'internet gourmand » semblent actualiser et complexifier les rationalités du mangeur. Il s'empare de ces médiations pour s'informer au plus près, tracer les origines des produits, géolocaliser des restaurants, gérer ses approvisionnements, suivre des 
cours (mooks, www.marmiton.org) mais aussi pour se faire livrer, gérer ses recettes de cuisines en ligne, donner son avis et ses commentaires (sur des lieux, des marques, des chefs : Yelp, Food Reporter, etc.), évaluer des plats, se mettre en scène (cuisinant, cultivant, mangeant,...), inviter à dîner des inconnus, préparer des dîners de quartier, réserver un chef à domicile, demander des conseils en termes de régime ou d'allergie (l'alimentation est un des sujets les plus en vogue sur les réseaux sociaux). Autant d'intermédiations numériques qui remettent en cause les modèles traditionnels (sur lesquels se reposent les acteurs du secteur alimentaire), sans pour autant mettre à mal les commensalités ré-émergentes. Ces usages numériques, s'ils questionnent de multiples manières la légitimité et la crédibilité des chefs, ouvrent aussi à de nouveaux rituels de ré-enchantement de l'alimentation, actualisent de nouvelles formes de curiosité gourmande et alimentaire.

Le mangeur se recentre sur ses pratiques. Il se prend ainsi en photo mangeant, photographie ce qu'il mange (food porn) et fait circuler ces images via les réseaux sociaux ou des blogs culinaires, véritables espaces de confessions alimentaires. Il $\mathrm{y}$ met en scène ses préférences alimentaires, parfois compulsivement, mais aussi, plus rarement, ses ambitions de 'chef amateur'. Il s'y affirme de plus en plus averti, évaluant à tout moment plats, restaurants, produits (via un like, un commentaire, un vote, un jugement). Le mangeur y exprime de manière profane son avis et endosse un rôle d'expert plus ou moins improvisé, sans avoir à justifier de compétences ni même à se soucier des répercussions de ce qu'il dit, écrit, signifie. Sa place change et fragilise donc les médiations en place.
Les médiations numériques participent donc à faire évoluer les pratiques alimentaires mais aussi le rapport aux organisations et aux institutions ; elles bousculent la place du chef, qui y perd en aura stable ce qu'il y gagne en résonance médiatique. Celui-ci doit et devra de plus en plus prendre place face à des consommateurs plus matures, qui demandent des comptes, vont chercher l'information, vérifier, collecter des avis, y compris sur des sujets que l'on disait experts (comme le développement durable, dans toutes ses dimensions). Des consommateurs 'connectés' qui éprouvent la parole publique, préemptent le sujet de l'alimentation, relayent et/ou adhérent en ligne à des initiatives citoyennes locales (dénonçant l'hyperconsommation, les inégalités alimentaires, etc.) et mettent à l'épreuve les récits marchands (mouvements anti-marques).

Progressivement le mangeur contemporain devient acteur à part entière des transformations des modes d'alimentation, prenant place dans l'arène des débats qui voit déjà s'affronter et/ou collaborer, dans de complexes jeu d'alliances, scientifiques, pouvoirs publics, militants écologistes. Ainsi le mangeur des villes prend la parole, dénonce une approche physiologiquement utilitaire de l'alimentation, s'investit dans les projets d'éco-cités. Il s'arme par l'information, s'invite dans les débats publics, interpelle et peut boycotter ou buycotter dans la perspective d'un consumérisme critique. On parle ainsi de citoyenneté et de démocratie alimentaires.

\section{Les engagements citoyens et habitants : entre résistances mondiales et fiertés locales}

$\mathrm{Au}$ fil de ces lignes, nous comprenons qu'au- 
delà d'une mutation des comportements et des pratiques alimentaires se jouent des changements de plus grande ampleur. Les grandes crises alimentaires (de la vache folle à la viande de cheval, en fait inoffensive en termes sanitaires) ont amplifié les peurs et éveillé des fantasmes (souvent attisés par les médias). Les émeutes de la faim et les innombrables luttes contre les inégalités alimentaires et la malnutrition ont secoué les consciences. En reprenant la main sur son alimentation et son devenir alimentaire le mangeur s'engage, politise sa relation à ce qu'il mange, revendique d'autres règles, normes et valeurs.

Par ailleurs, s'amplifient et se diversifient les réponses données par les habitants aux dispositifs publics mis en œuvre autour de l'alimentation durable, de l'agriculture urbaine et des solidarités alimentaires. Ainsi en est-il pour le permis de végétaliser qui s'inscrit dans la continuité des initiatives autour des jardins partagés, invitant à redécouvrir fruits, légumes, espèces sauvages au pied de son immeuble 9 .

\section{Le mangeur " citoyen "}

Ces dernières années ont vu se déployer une grande diversité de contestations citoyennes : que cela soit les résistances des habitants face à l'installation de fast foods dans les centres villes historiques, de centres commerciaux géants au détriment des commerces de bouche traditionnels ou les combats pour des solidarités alimentaires, pour la défense du monde agricole ou l'agriculture urbaine.

Du Do it yourself individuel (cultiver herbes et plantes aromatiques, fruits et légumes, de manière multiple ou collective, notamment dans la rue, avec le « permis de végétaliser ») aux militantismes organisés, le mangeur s'engage donc de manière offensive et concertée pour le développement alimentaire durable : ainsi en défendant les circuits courts, en encourageant les initiatives locavores, en réclamant des cantines scolaires bio, en dénonçant les dérives du système de production alimentaire mondialisée (OGM, pesticides, malbouffe, alicaments...). Les enjeux ne sont plus tant nutritionnels que politiques, sociologiques, écologiques : les États généraux de l'alimentation, l'élaboration des politiques alimentaires (qui, pour rappel, ont pour ambition d'installer le dialogue entre les différentes catégories d'acteurs sociaux, de mettre en débat les choix politiques qui accompagnent la modernité alimentaire pour construire ou reconstruire $d u$ consensus) s'appuient ouvertement sur ces militantismes renouvelés, ce passage de l'individuel au collectif (exemple du mouvement vegan qui ne signifie plus seulement une peur mais aussi un désaccord et une revendication).

Le mouvement slow food ${ }^{10}$ est certainement le plus visible de ces mouvements, le plus mondialisé. Organisé autour de centaines de conviviums (composés de militants locaux et parfois de chefs) constituant un réseau dense, dédié à la sauvegarde de la diversité alimentaire (sans combattre pour autant la globalisation), au maintien des petits producteurs qui cultivent des produits de qualité (notamment des produits 'sentinelles') et à leur accompagnement dans la conquête de nouveaux marchés, ce mouvement participe à cette conscientisation des citoyens et à cette structuration de mobilisations locales et internationales. Construit autour des enjeux d'une 
nourriture engagée, d'une défense de la biodiversité et d'une éthique du 'bien manger', il arme le citoyen pour la réflexion sur ce qu'il mange, sur le fonctionnement du système agro-alimentaire actuel, sur les différentes options possibles quant à l'évolution de notre mode d'alimentation.

Bien d'autres initiatives citoyennes illustrent cette défense d'une alter-consommation (à des échelles internationales, nationales ou locales) ainsi :

\section{- Les Incroyables comestibles (https://lesin-} croyablescomestibles.fr) : un mouvement citoyen de bien commun (en open source), d'origine anglaise mais présent dans 25 pays dans le monde, non marchand et s'énonçant comme 'a-politique' (au sens partisan du terme). Centré sur l'agriculture urbaine participative, il s'adresse à tous et se donne comme principal enjeu de « nourrir l'humanité de façon saine pour l'homme et la planète, localement en suffisance ». Il invite les habitants à planter des légumes en villes, partout, cherchant, par la nourriture (comme facteur d'unité et de convivialité) à reconnecter les gens entre eux et à les reconnecter à la terre nourricière. La structure française facilite donc le développement du mouvement à l'échelle nationale et locale ;

- Les Urbainculteurs (www.urbainculteurs.org) : un organisme à but non lucratif (proposant des services gratuits et marchands) dont la mission est de promouvoir le jardinage et l'agriculture urbaine et qui œuvre (depuis 2009) au développement d'une agriculture urbaine productive et innovante au Québec et ailleurs (conception collaborative de potagers urbains, sensibilisation aux questions citoyennes, engagements contre les déserts alimentaires, les îlots de chaleur, etc.) ;
- Horizon alimentaire et Anges gardiens (http :// horizonalimentaire.fr/angesgardiens.) : cette initiative associative, encore confidentielle, intégrée à l'Éco-pôle alimentaire de la région d'Audruicq ${ }^{11}$, vise l'insertion, l'éducation permanente, le développement de pratiques sociales et d'activités liées à l'alimentation. Le dispositif est complété par l'Ambassade du bien vivre, un espace de partage du savoir culinaire et vivrier.

\section{La consommation collaborative : entre pragma- tisme et militantisme}

Progressivement le mangeur des grandes villes s'inscrit dans une société basée sur le partage, la collaboration et la contribution ${ }^{12}$. La consommation collaborative regroupe un ensemble de pratiques de consommation alternatives tentant de répondre aux attentes des consommateurs en matière de santé et de respect de l'environnement et cherchant à augmenter l'usage d'un bien ou d'un service par le partage, le troc, l'échange, la vente et entre particuliers. Elle privilégie l'usage sur la propriété, le partage sur l'achat de biens et de service et se traduit donc par des pratiques de co-utilisation, de co-élaboration, de cohabitation, de mise en réseaux. Dans le cas de l'alimentation, la consommation collaborative témoigne bien d'une nouvelle étape dans ce réinvestissement citoyen, dans ces combats autour du 'manger durable' : polymorphe, et de plus en plus structurée, elle peut à terme bouleverser en profondeur les modèles de production, de fabrication, de distribution, de consommation alimentaires et l'idée même de lien social autour du partage alimentaire (dont les formes se diversifient, se complexifient jusqu'à l'insolite). Elle se veut une réponse plurielle à la défiance vis-à-vis des 
industries alimentaires mais aussi à une crise sociale et culturelle plus large. Et cette réponse (visant la reconquête d'une confiance) se traduit par une multiplication de services collaboratifs localisés ou en réseau : si le mode de vie collaboratif avait l'image d'une consommation locale et située, avec l'usage d'internet, il s'ouvre à un nombre croissant de personnes ; si certains services coopératifs sont anciens et furent longtemps confidentiels (à l'exemple des $\mathrm{AMAP}^{13}$ ), le recours aux médiations numériques leur donne aujourd'hui une visibilité et une capacité d'accès inédits. Internet et les réseaux sociaux sont, en effet, au cœur de cette consommation collaborative qui voudrait devenir un modèle dominant et non plus contestataire. Les modes de communication numérique qui défendent l'émergence d'une culture du « nous » participent non seulement à réinventer ce que l'on consomme, mais également la manière dont on les consomme. Favorisant un renouvellement des formes de distribution et d'échange, ils participent à réorganiser progressivement, et l'air de rien, nos vies ${ }^{14} \ldots$ et surtout celle des jeunes générations, 'hyperconnectées' et semblant se retrouver dans les valeurs de l'économie collaborative, dans la logique d'échange entres pairs, dans le partage de l'information. Illustrons ces intermédiations collaboratives autour de l'alimentation par l'esquisse d'une typologie :

- Des sites de commandes en ligne entre habitants : ainsi Tous cuistots (blog.touscuistots.fr) «...qui permet à des cuisiniers amateurs (ou pro) de vendre leurs plats cuisinés à des gourmands ne sachant pas ou n'ayant pas le temps de faire la cuisine»; Super marmite (blog.super-marmite.com), un réseau social géolocalisé qui « révèle les plats maison qui mijotent 'à deux pas de chez nous'» ;

- Des sites de mises en contact de mangeurs : ils répondent de même à une demande de lien social autour du repas (mis à mal par la grande distribution), de partage de plats faits maisons ou de repas dits collaboratifs (ainsi Vizeat, https :// fr.vizeat.com, une plateforme de repas collaboratifs qui permet d'accueillir des étrangers à sa table, habitant ou touriste) ;

- Des sites de mises en contact consommateurs et producteurs : dans le sillage des AMAP, se diversifient les groupements de consommateurs pour l'achat de fruits et de légumes locaux afin de « réduire leur exposition au risque»; citons Chez vos producteurs (www/chezvosproducteurs. $f r$, plateforme de vente directe de produits fermiers avec les producteurs) ou la Ruche qui dit oui (https ://laruchequiditoui.fr ), une place de marché en ligne ;

- Des sites de trocs et d'échange qui vont du partage de jardins ou de récoltes de produits du jardin au partage et troc d'outils, de compétences, de disponibilités, etc. : Ca vient du jardin (wwwcavientdujardin.com), le Potiron.fr, Troc légumes (www.trocalimentaire.com) ;

- Des sites invitant à l'occupation alimentaire des espaces publics : outre les Incroyables comestibles (Incredible Edible), d'autres occupations informelles dans les espaces publics, dédiées à l'alimentation, se préparent dans le monde via l'intermédiation numérique (du Canada au Brésil ou à l'Inde) ;

- Des forums d'information : ils proposent des 
discussions autour des questions de qualité, sécurité, traçabilité mais aussi autour de recettes, de conseils nutritionnels, d'idées pour cuisiner autrement (le Nantes food Forum, le réseau Colibris) ;

- Des initiatives décloisonnant les mondes : l'Open food fondation via l'Openfood network (https :// openfoodnetwork.org), une infrastructure open source qui permet à tout acteur, individu, communauté, groupement d'achats à but non lucratif, coopérative, start-up travaillant sur l'alimentation locale, de gérer leurs opérations quotidiennes mais aussi de coopérer, « chacun à sa manière ».

Autant de sites et d'applications qui cherchent à mieux cadrer la provenance des produits, à ouvrir de nouvelles possibilités de distribution aux producteurs, à permettre aux individus d'acheter en direct aux producteurs et donc qui mettent au centre les intermédiations numériques pour faciliter la circulation de l'alimentation, témoignant de la capacité des individus à s'organiser en dehors des institutions et des organisations en place.

L'engagement citoyen est ici polymorphe et offensif, partant du postulat que les problèmes générés par les industries et plus avant par le système alimentaire en place ne seront résolus que par des réponses concrètes, alternatives et localisées. Mais, au-delà des motivations citoyennes d'achat responsable, la diffusion des pratiques de consommation émergentes semble être portée aussi par des motivations plus individuelles (volonté de réenchanter la consommation, de consommer des produits alimentaires de meilleure qualité, plus adaptés à notre santé). Consommation et militantisme dialoguent 'sur le fil'.

\section{L'alliance en devenir des chefs et des habitants?}

L'ensemble de ces pratiques bouscule la relation chef et mangeur. Le premier pour être audible, visible et crédible doit sans cesse être en veille, s'ajuster, donner des preuves de son talent, de son originalité, de sa valeur, anticiper tendances et critiques et réviser en continu sa communication. En cela, nous pouvons nous demander si l'évolution de cette communication (du culinaire à l'alimentaire), sa sortie croissante de la cuisine ne le protègeraient pas en déplaçant la scène de sa crédibilité sur d'autres scènes ? Ne lui offriraient-elles pas l'occasion de construire sa réputation non seulement sur la base d'expertises étendues mais aussi de 'valeurs' contemporaines (collaboratives, citoyennes, démocratiques)? Le chef, ayant pris conscience de la défiance des consommateurs, ne communiquerait-il pas sur 'autre chose' que sa seule cuisine pour endosser les valeurs du temps, pour rejoindre les combats consensuels et redéfinir de manière incontestable la gastronomie comme une réponse à la crise planétaire. L'excellence culinaire ne suffisant plus au mangeur contemporain, le chef se construit comme leader ne cherchant plus seulement à prendre une position centrale voire hégémonique dans la hiérarchie des métiers de la gastronomie mais un positionnement transversal et médiateur dans 'l'écosystème alimentaire'.

\section{Demain : un chef collaboratif et passeur de territoire?}

Nous avons donc pointé quelques traits saillants du mangeur contemporain, moins passif ou malinformé que ne le laissaient entendre les montées au créneau salvatrice des chefs étudiées dans le 
précédent article. Dès lors, nous pouvons interroger plus avant l'adéquation de ces engagements affichés par les chefs avec des attentes et des comportements effectifs des mangeurs.

\section{Chefs 2.0 : quand la gastronomie devient connectée}

Les figurations étudiées semblent parfois quelque peu dérisoires ou à courte vue quand 1'on constate la rapidité des mutations des pratiques tant alimentaires que numériques des jeunes et des adultes de demain. Certes, certains chefs déploient des stratégies relationnelles en ligne 'à $360^{\circ}$ : ils twittent, lancent des sites responsive, partagent des photos de leur brigade sur les réseaux sociaux, postent des selfies, revendiquent fans et followers ${ }^{15}$. Certains alimentent déjà la toile à coups de hashtags et de filtres Instagram et deviennent 'incontournables'. Très présents sur les médias sociaux (leviers d'influence et de notoriété pour les chefs qui envisagent une relation 'intime' avec les socio-nautes), nombre d'entre eux ont recours à des agences de communication orientées social media qui définissent pour eux une ligne éditoriale 'conversationnelle' ${ }^{16}$. Mais en poussant l'analyse, les engagements énoncés en ligne par les grands chefs précédemment étudiés semblent, au vu des exemples d'émancipations consommatrices et citoyennes évoquées, relever plus d'une stratégie performative que d'une recherche (concrète et située) de relation adaptée et renouvelée avec les mangeurs.

Pourtant, au niveau local, l'échelle de proximité, la donne évolue rapidement. Les actes de communication se font plus situés, 'civiques', de longue portée. En effet, les chefs (anonymes pour bon nombre) s'associent aux politiques de développement territorial (culturel, touristique, patrimonial) : ils participent à l'actualisation des inventaires des traditions alimentaires et culinaires des territoires (et notamment ceux des « sites remarquables » du goût ${ }^{17}$ ), sont invités à renouer et consolider le lien ancestral existant entre producteurs, artisans, commerçants et à s'inscrire dans des stratégies de marques touristiques destinées à valoriser les destinations et les patrimoines gourmands... Mais ils se font aussi, certes parfois de manière ambivalente, plus proches des habitants en devenant passeurs culturels et patrimoniaux, via divers dispositifs et de médiations...

\section{Le chef, médiateur culturel et patrimonial des destinations gourmandes}

Nombre de collectivités locales demandent ainsi aux chefs de s'inscrire concrètement dans la gouvernance culturelle des territoires en participant aux instances touristiques et patrimoniales dédiées à leur labellisation, à leur mise en marque et à leur évènementialisation. Quelques exemples illustreront cette tendance :

- À Saint-Jean-de-Monts, l'OPACT (Office des Patrimoines Culinaires des Terroirs, défendant la cuisine montoise) qui regroupe chefs, ethnobotanistes, zoologues, producteurs, experts du patrimoine matériel et immatériel. Créé en 2017 au sein de l'OPCI (Office du Patrimoine Culturel Immatériel, https ://opci-ethnodoc.fr), c'est un groupe (cluster) d'acteurs des secteurs du patrimoine, de la gastronomie et du tourisme qui a pour but de 'révéler' les identités culturelles relatives à la cuisine traditionnelle, en lien 
avec les filières économiques et touristiques des territoires. Le chef y tient une place importante ;

- Dans le cadre de la création des quatre Cités de la Gastronomie (Tours, Dijon, Lyon, ParisRungis) autour du Repas Gastronomique des Français (labellisé en 2010 patrimoine mondial par l'UNESCO), des chefs intègrent en tant que 'personnalités qualifiées' leurs conseils scientifiques, culturels et techniques ou bien les associations qui en portent le projet (ainsi Tours Cité internationale de la gastronomie en Val de Loire) ; ils y proposent des programmations festives, y ouvrent leurs réseaux locaux (l'ensemble des acteurs de la chaîne alimentaire) pour susciter des initiatives culturelles et patrimoniales ;

- Plus largement encore, le déploiement des SAT (Systèmes Alimentaires Territoriaux) ${ }^{18}$ amène les chefs à porter des démarches collectives, à défendre la relocalisation des acteurs de l'alimentation dans une perspective de cohérence, de cohésion et de plus grande autonomie alimentaire. Envisagés comme forces de proposition et d'animation, ils s'associent ainsi, de manière instituée, aux associations de producteurs, aux transformateurs, aux chambres consulaires, aux artisans locaux... en tentant, ici aussi, d'impulser des démarches transversales.

Le chef se fait (nous l'avions abordé dans le précédent article) 'ambassadeur' à l'international (gastro-diplomatie) mais aussi à l'échelle locale, au plus près des terroirs, des savoirs et des savoirfaire locaux, des mémoires des habitants. Son action territoriale recouvre une diversité croissante de formes : participer à l'élaboration des politiques alimentaires locales (œuvrant pour la transition alimentaire, la démocratie et la souveraineté alimentaire locales, une relation renouvelée à la nature) ; améliorer l'attractivité et la notoriété alimentaires, culinaires et gastronomiques du territoire ; œuvrer ainsi à la patrimonialisation de l'alimentaire et du gastronomique (notamment via la candidature aux labels patrimoniaux) ; défendre les identités commerciales des territoires et développer leur 'identité' culinaire mais aussi interpeller les mémoires vivantes des habitants ou encore aider au renforcement de l'éducation et de la culture alimentaires...

Ainsi, en Vendée, l'OPACT encourage les habitants de Nantes et de ses environs à s'associer à ses démarches : un chef, le toqué Yvon Garnier (membre de l'Office), veut lancer un Comité de soutien pour la création d'un Conservatoire des arts culinaires pour représenter l'identité culinaire locale ${ }^{19}$ et semble rencontrer l'oreille tant des élus que des habitants 'partants' pour l'aventure ; les chefs du Pays de Monts vont, quant à eux, à la rencontre des habitants envisagés comme détenteurs de recettes issues de la transmission familiale ou coopérative. Avec eux, ils dressent des inventaires (de recettes ou d'ingrédients), documentent historiquement et anthropologiquement les sources recueillies.

Au niveau local, l'engagement des chefs semble donc voué à de beaux lendemains. Et le déploiement des quatre Cités de la Gastronomie, énoncées comme des 'Centres Pompidou de la Gastronomie', des espaces pluridisciplinaires dédiés à la fois aux produits, aux savoir-faire et aux métiers ainsi qu'à la question de l'innovation, devrait conforter leur place et les amener à devenir plus encore des forces de propositions et 
d'initiatives inédites (actions de labellisation, de conception évènementielle, de mise en tourisme, d'éducation, de transmission des savoirs mais aussi d'innovations culturelles et artistiques).

L'investissement pluriel des chefs dans les dispositifs mis en place par les institutions locales s'éclaire à la fois par ces mutations institutionnelles (plus ou moins ordonnées et articulées) et par ces changements d'attente et de comportements des mangeurs. Mais les médias généralistes n'en rendent que partiellement compte en se focalisant plus sur la figure du 'grand' chef que sur les diverses médiations initiées ou auxquelles s'associent les chefs moins médiagéniques. Certaines vidéos postées par des comités départementaux ou régionaux du tourisme (diffusées à travers les réseaux sociaux et les canaux 'grand public') semblent commencer à leur donner une place, mettant parfois en récit leurs actions au quotidien, revenant à leurs savoir-faire mis en partage et inscrits dans une histoire. Le chef, cet innovateur appelé à réveiller les territoires de leur torpeur gastronomique, de leurs routines culinaires, deviendrait alors un incontournable acteur «multitâches» et un levier central dans la redynamisation des systèmes alimentaires, culinaires et gastronomiques locaux.

\section{Le restaurant : du marqueur territorial au laboratoire connecté?}

Nous n'avons jusqu'ici aucunement évoqué les restaurants, car la construction des figures abordées dans le premier article (le chef engagé dans la Cité, dans la nation et à l'international) invisibilisait ces espaces marchands, leurs liens historiques et consubstantiels au chef, pour ins- crire celui-ci à des échelles élargies (le terroir, le territoire, la planète)... Mais, avant de conclure, évoquer ce lieu permet de conforter l'hypothèse d'une reterritorialisation actuelle de cette figure évolutive du chef cuisinier.

Dans le cadre des stratégies de développement territorial des patrimoines (alimentaires, culinaires et gastronomiques) qui se développent un peu partout en France, ce lieu cristallise enjeux, projections et appropriations, entre « lieu carrefour » et « vitrine identitaire $»^{20}$. Il s'énonce parfois comme une vigie pour l'ensemble des lieux alimentaires et gourmands du territoire (fermes, potagers, jardins légumiers, marchés et lieux marchands...) : en en mettant en scène les attributs et les produits alimentaires phares, en sémiotisant leurs qualités "identitaires ", il se patrimonialise. Il s'évènementialise aussi : scène de pop up éphémères, de performances culinaires ludiques, de dégustations commentées; lieu d'apprentissage (via des ateliers), d'expériences 'sensorielles' (qui s'énoncent, avec les accents enchanteurs des logiques de marque, comme 'inédites', 'insolites', la composante gustative et gastronomique se mêlant alors aux autres sens) ou encore d'expérimentations sociales, culturelles, technologiques (auto-désignation des restaurants comme ' $l a b s$ '), le restaurant de demain s'adapterait donc tout à la fois à l'injonction patrimoniale et à celles de l'expérimentation et de l'innovation, sortant ainsi d'un certain isolement et de certaines solitudes.

«L'idée serait de créer un lieu qui redonnerait de l'importance à ce qui se joue en salle. Une table qui réinventerait la commensalité. Le restaurant conçu non plus seulement comme le plateau où 
s'exprime la créativité glorieuse d'un seul, où se vit une expérience sensorielle individuelle, mais comme lieu d'un rapport à l'autre, et d'une possible aventure collective $»^{21}$.

\section{Conclusion}

Pour conclure, rappelons combien l'alimentation est le symbole d'un choix de société et apparaît aujourd'hui comme un passionnant objet pour ressaisir les mutations du monde, pour penser le devenir de nos sociétés démocratiques. Si elle devient une affaire publique centrale, une scène d'expressions contradictoires, de revendications (tant par rapport aux pouvoirs publics qu'aux acteurs marchands de l'agro-alimentaire), de dissensions et de désaccords (par rapport au devenir alimentaire de la planète), ses réécritures et ses mises en récit passent par un intense travail symbolique et éditorial, demandant ordonnancement et approfondissement : le chef mais aussi l'agriculteur (notamment urbain), l'agronome, l'artisan de bouche, le maraîcher, le jardinier deviennent ainsi des pôles d'aimantation sociale et des emblèmes incarnant ces mutations - et ceci à différentes échelles ( mondiale, nationale, régionale et locale).

Ainsi, à l'origine instrument au service de la diplomatie, la gastronomie nationale est dorénavant promue par la diplomatie qui s'emploie à défendre les patrimoines culinaires des États, envisage leur valorisation comme visée et non pas seulement comme simple outil des relations diplomatiques. Cet essor diplomatique s'affirme avec la mise en valeur des cultures et des patrimoines gastronomiques par l'UNESCO mais aussi avec les diverses actions de défense de nos exportations. Le grand chef étoilé est ainsi passé du statut de petit entrepreneur, puis d'homme d'affaires (monétisant son savoir-faire) à celui d'ambassadeur des cultures alimentaires (Alain Ducasse articulant 'magistralement' cette double casquette). Mais si la plupart des engagements affichés, étudiés au fil de ces deux articles, semblent encore souvent participer d'une stratégie ostentatoire et grégaire (le grand chef œuvrant à l'effort de diplomatie culturelle, dans un contexte économique et culturel en tension), l'échelon local ouvre d'autres perspectives.

C'est au niveau local que les engagements des chefs vers les mangeurs que nous sommes (habitants ou visiteurs) se font plus situés, plus concrets et moins ostentatoires ou simplement encore faiblement médiatisés. La relation de confiance se complexifie et demande de la part des chefs locaux une grande adaptabilité : elle ne se fonde plus sur la seule qualité des plats mais sur les garanties écologiques, durables, culturelles qu'ils peuvent donner. Leurs relations aux habitants et aux citoyens mais aussi aux touristes sont donc vouées à évoluer.

Au fil de ces deux articles, nous avons volontairement laissé ouverte la définition du chef (cuisinier), évité d'établir une claire distinction entre 'grand chef', 'chef étoilé' et simple 'chef cuisinier', mis de côté les définitions professionnelles... tant les représentations envisagées nous semblaient jouer sur les limites et déplacer les hiérarchies. Par le biais de cet éventail de figures nous avons pu porter un regard inédit sur la construction symbolique des politiques alimentaires publiques. Plutôt que de subir les mutations techniques, culturelles et sociales que connaît la 
question de l'alimentation, il semble que le chef veuille faire bouger les lignes et le faire savoir : jouant de sa réputation, de sa célébrité, voire de sa renommée, il repositionne et redéfinit son influence en tant qu'acteur du changement sociétal, porte-voix des valeurs du développement durable, du mieux vivre alimentaire, redéfinissant la gastronomie comme étant en mesure de résorber la crise. 'Grand' ou anonyme, le chef est en train de redessiner, via les médias, son avenir et sa place dans la société, dans la ville, dans le quotidien de chacun.

\section{$R \cdot E ́ \cdot F \cdot E ́ R \cdot E \cdot N \cdot C \cdot E \cdot S$}

ALKON Alison Hope, GUTHMAN Julie, The new food activism, opposition, cooperation and collective action, University of California Press, 2017.

ASCHER François, Le mangeur hypermoderne. Une figure de l'individu éclectique, Éditions Odile Jacob, 2005.

BARRÈRE Christian, « Patrimoines gastronomiques et développement local : les limites du modèle français de gastronomie élitiste ", in Mondes du Tourisme, $\mathrm{n}^{\circ} 3,2013$, p. 15-36.

CHARAUDEAU Patrick, « Le charisme comme condition du leadership politique ", dans Les recherches sur les publics en Sciences de l'Information et de la Communication, in Revue Française des Sciences de l'information et de la communication, $\mathrm{n}^{\circ}$ 7, 2015.

KERANDAL Maylis de, Un chemin de table, collection Raconter la vie, éditions du Seuil, 2016. FISCHLER Claude :

- L'Homnivore, Paris, Odile Jacob, 1990.

- Les Alimentations particulières : mangeronsnous encore ensemble demain ?, Paris, Odile Jacob, 2013.

MILLLER Jeff, DEUTSCH Jonathan, Food Studies, an introduction to research méthods, Bloomsburry, Oxford, New York, 2009.

PAGÈS Dominique :

- « Figures du chef cuisinier. Première partie : de la créativité culturelle à l'engagement sociétal et public », in Quaderni, n 94 ;

- «Permis de végétaliser », 1, 2, 3 : http :// food20.fr/urbainculteurs-avez-permis-de-vegetaliser/; http ://food20.fr/permis-de-vegetaliser2-de-ville-verte-a-ville-vivriere/; http ://food20. 
fr/permis-de-vegetaliser-3-semiotisation-nourriciere-de-nos-villes/; site de recherche Food Lab 2.0, 2017.

POULAIN Jean Pierre :

- Sociologies de l'alimentation, nouvelle édition augmentée, Paris, PUF, 2017.

- L'homme le mangeur et l'animal, Actes du colloque OCHA, 2007.

POULAIN Jean Pierre, en collaboration avec Jean-Pierre CORBEAU, Penser l'alimentation, Entre imaginaire et rationalités, Privat, première édition 2002, 210 pages, seconde édition 2008. REVUE Espaces, Tourisme, Loisirs, dossier «Restauration et tourisme », $\mathrm{n}^{\circ} 338$, octobre 2017 (https ://www.tourisme-espaces.com/doc/ tourisme-loisirs-culture.html).

COLL. Mondes du tourisme, " Gastronomie et développement local », n7, Juin 2013 (https :// www.tourisme-espaces.com/doc/8840.mondestourisme-gastronomie-developpement-local. html)

SCHLIENGER Jean-Louis, Manger au XXI siècle... pas si simple! - Entre désarrois et plaisirs alimentaires, Belvédère (Éditions du), Paris, O. Jacob, 2016.

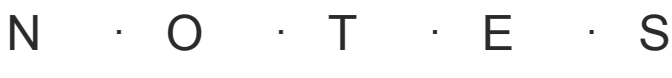

1. René Redzepi, chef de file de la cuisine nordique, est sans doute l'un des chefs qui orchestrent le plus offensivement cette fabrique de la réputation de soi. Nommé ambassadeur de la cuisine nordique, il tient un discours militant, un brin naturaliste, tout en faisant fructifier ses affaires. Voir (site Foodlab 2.0) articles de l'auteur sur le sujet.

2. «Figures du chef cuisinier. Première partie : de la créativité culturelle à l'engagement sociétal et public », in Quaderni, n 94, automne 2017.

3. www.ouest France.fr, 27 septembre 2017.

4. Patrick Charaudeau, « Le charisme comme condition de leadership politique », op.cit.

5. Se référer à la bibliographie.

6. www.lemangeur.ocha.com, l'Observatoire CNIEL des habitudes alimentaires.

7. Voir les données fournies et les analyses des croyances de notre époque proposées par le livre de Jean Louis Schlienger, Manger au XXI siècle, pas si simple, entre discours et plaisirs alimentaires, Éditions du Belvédère, 2016.

8. Voir le subtil ouvrage de Maylis de Kerandal, Un chemin de table, collection Raconter la vie, éditions du Seuil, 2016. 9. Voir l'analyse des actions de la Ville de Paris dans ce sens, au fil de trois articles sur ce permis de végétaliser : http://food20. fr/urbainculteurs-avez-permis-de-vegetaliser/; http://food 20. $\mathrm{fr} /$ permis-de-vegetaliser-2-de-ville-verte-a-ville-vivriere/; http://food20.fr/permis-de-vegetaliser-3-semiotisationnourriciere-de-nos-villes/.

10. Slow Food : « Pour une alimentation bonne, propre et juste », www.slowfood.com, http://slowfood.fr.

11. Un centre ressource et de développement de nouvelles activités agricoles et d'appui aux systèmes alimentaires locaux.

12. Voir le collectif Ouishare (www.ouishare.net, association fondée en janvier 2012 à Paris, devenue rapidement un acteur international de premier plan dans le domaine de l'économie 
collaborative).

13. AMAP: Association pour le maintien d'une agriculture paysanne (www.amap.idf.org).

14. Voir les nombreux articles publiés dans ce sens sur les sites et portails : Foodlab.2.0 (www.food2.0.fr), Atabula (www.atabula.com), Alimentation générale (www. alimentation-generale.net).

15. On parle alors de 'marketing relationnel' (l'ensemble des actions marketing qui visent à établir une relation continue, renforcée et enrichie avec le consommateur afin de fidéliser et d'augmenter continuellement son équipement et sa consommation). Le marketing relationnel vise à développer les relations en dehors même de moments de consommation ou d'achat.

16. Ainsi, les agences Eliophot (www.eliophot.com), Melchior (www.agencemelchior.com), Alioze (www.alioze.com).

17. Les sites remarquables du goût: www.agriculture.gouv. fr-sites.remarquables-du-goût-desescapades-made.france)

18. Voir sur le sujet le site du CERD (Centre Ressource du Développement durable).

19. www.ouest France.fr, 19 décembre 2016.

20. Voir la Revue Espaces, Loisirs et Tourisme, n 338.

21. Un chemin de table, op.cit. p. 102. 